EPJ Web of Conferences 37, 01006 (2012)

DOI: $10.1051 /$ epjconf/20123701006

C Owned by the authors, published by EDP Sciences, 2012

\title{
Recent results from LEPS and prospects of LEPS II at SPring-8
}

\author{
M. Niiyama ${ }^{\mathrm{a}}$
}

Department of Physics, Kyoto University, Kyoto, Japan

\begin{abstract}
SPring-8/LEPS is a GeV photon beam facility for hadron physics. The linearly polarized photon beam is produced by backward-Compton scattering of laser photons from $8 \mathrm{GeV}$ electrons. We have studied photoproduction of hadrons which contain strange quarks. In this article, firstly, we report one of the recent results from LEPS, the $\kappa(800)$ meson search in the $\gamma p \rightarrow K^{* 0} \Sigma^{+}$reaction. Secondly, the status of the construction of a new $\mathrm{GeV}$ photon beam line LEPS II is described.
\end{abstract}

\section{$1 \kappa(800)$ meson search in the $\gamma p \rightarrow K^{* 0} \Sigma^{+}$reaction}

\subsection{Introduction}

In the quark model, scalar mesons are described as the $L=1$ excitation of $q \bar{q}$ pair. However, the assignments to the experimental data are not clear. For example, the Particle Data Group states that identification of scalar mesons is a "longstanding puzzle" [1]. The existence of $a_{0}(980)$ and $f_{0}(980)$ is firmly established, but its internal structure, $q \bar{q}$ state or $q q \bar{q} \bar{q}$ state, is under discussion. The lightest unflavored scalar meson is assigned as $f(500)$ [1], and considered to be chiral partner of pion, $\sigma$. On the other hand, the $J^{P}=0^{+}$partner of the kaon, $\kappa$, is not established firmly. The difficulty with establishing the existence of the $\sigma$ or $\kappa$ mesons is that their resonance widths are very broad, (about $400 \mathrm{MeV}$ or wider). Hence they are difficult to observe in partial wave analysis of meson scattering data. The Fermi lab E781 group reported the existence of $K \pi$ resonance with $J^{P}=0^{+}$in the $D$ meson decay [2]. Recently, another evidence has been found from fits to Dalitz plots of final states in $D$-meson decay [3]. However, because those analysis doesn't show a clear mass peak, additional evidence is desired before the $\kappa$ meson can be firmly established.

We report on the measurement of linear polarization observables of $K^{* 0} \Sigma^{+}$photoproduction off proton at LEPS [4]. These observables, the spin-density matrix elements, are sensitive to meson exchange in $t$-channel reaction. At the SPring-8, the decay products of $K^{* 0}, K^{+} \pi^{-}$pairs, are detected at forward angles, where $t$-channel reaction is dominant. For the $\gamma p \rightarrow K^{* 0} \Sigma^{+}$reaction, the theoretical models at forward angles are rather limited. The $K^{0}$ exchange in $t$-channel occurs through the magnetic coupling of $K^{*}$ to photon. The hadronic coupling of the $K^{0}$ to the proton, $g_{K N \Sigma}$, is already measured from kaon scattering data [5]. Thus, the theoretical ambiguity is small for this mechanism. Exchange of a $K^{* 0}$ in the $t$-channel is suppressed, since only higher (non-spin-flip) multipolarities can contribute to this diagram [6]. The contact term is proportional to the vector meson charge, and vanishes for the neutral $K^{* 0}$ production. In addition to the mechanisms described above, a scalar meson exchange in the $t$-channel can contribute to $K^{* 0}$ photoproduction. By comparing the experimental data measured with two theoretical calculation, one with minimal $\kappa$ meson exchange and the other with substantial exchange, we can investigate the existence of the $\kappa(800)$.

The vector meson photoproduction with linearly polarized photons can be used as a parity filter, which constrain $J^{P}$ of exchanged particles in the $t$-channel. The polarization observables are measured from asymmetry in the distribution of azimuthal angle of decay products of the vector meson with

\footnotetext{
a e-mail: niiyama@scphys.kyoto-u.ac.jp
} 
respect to the polarization angle of the photon. The parity spin asymmetry [6], given in terms of the spin-density matrix elements by $P_{\sigma}=2 \rho_{1-1}^{1}-\rho_{00}^{1}$, is particularly sensitive to the role of $\kappa$ exchange. In the case of scalar $\kappa$ exchange, the parity spin asymmetry is positive, whereas calculations without the $\kappa$ (with pseudoscalar kaon exchange only) have negative parity spin asymmetry.

\subsection{Experiment}

The experiment was carried out at the SPring-8/LEPS facility in Japan. A linearly polarized photon beam is provided by the backward Compton-scattering of laser photons with $8-\mathrm{GeV}$ electrons. The energy of photon beam was in the range of 1.5 to $2.96 \mathrm{GeV}$. The photon beam was incident on a 15 $\mathrm{cm}$ liquid hydrogen target. The $K^{+}$and $\pi^{-}$particles were produced and momentum analyzed by the LEPS spectrometer [7]. To identify candidate events, a $K^{+}$track and a $\pi^{-}$track were selected from the reconstructed masses which are calculated using momenta and time of flight. The vertex of the $K^{+}$ and $\pi^{-}$tracks were required to be in the region of the $\mathrm{LH}_{2}$ target. The $K^{* 0} \Sigma^{+}$production events were selected by identifying a peak of $K^{* 0}$ and $\Sigma^{+}$in the invariant mass spectrum of $K^{+} \pi^{-}$system and in the missing mass of the $\gamma p \rightarrow K^{+} \pi^{-} X$ reaction, respectively. Fig. 1 shows an invariant mass spectrum of $K^{+} \pi^{-}$with $\Sigma^{+}$selection (a) and a missing mass spectrum of $K^{+} \pi^{-}$with $K^{* 0}$ selection (b). One can see clear peaks corresponding to $K^{* 0}$ and $\Sigma^{+}$. The decay angular distributions of $K^{+} \pi^{-}$were obtained in two reference frames, the Gottfried-Jackson (GJ) frame and helicity frame. Then the parity spin asymmetry was obtained from the spin density matrix elements, as shown in Fig.2. The red and blue points show the asymmetry calculated in GJ frame and helicity frame, respectively. The solid (dashed) line is the result of model I (model II) of Ref. [6] at $E_{\gamma}=2.5 \mathrm{GeV}$. Model I has almost no contribution from $\kappa$ exchange, whereas model II includes substantial $\kappa$ exchange. Clearly, the observed asymmetry favors the model II with the contribution of $\kappa$.
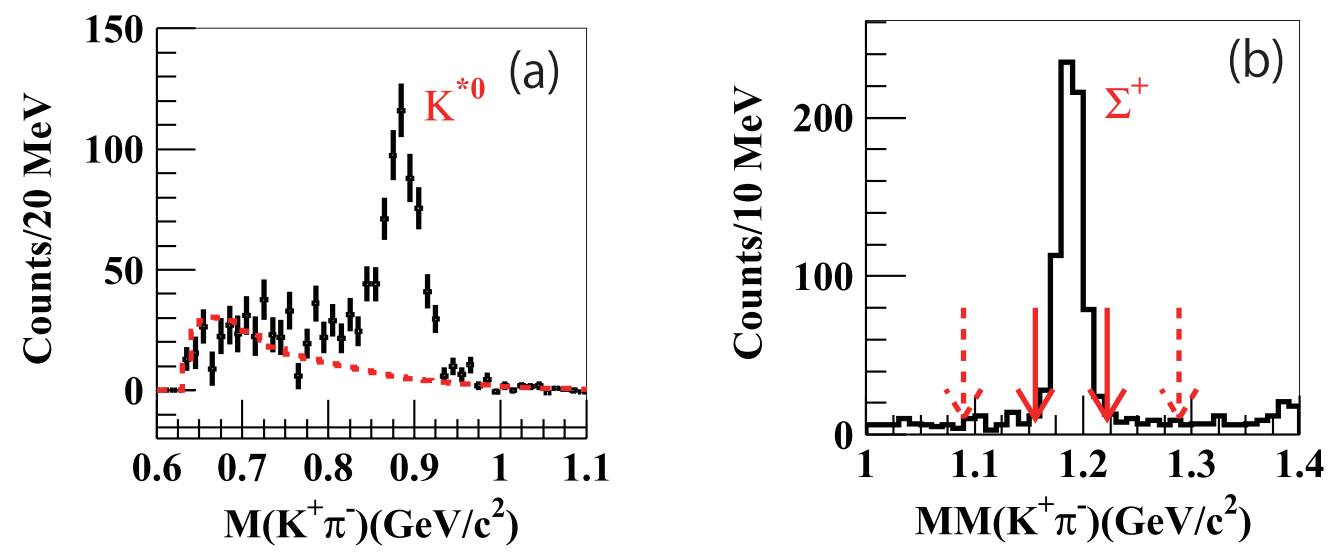

Fig. 1. Invariant mass spectrum of $K^{+} \pi^{-}$with $\Sigma^{+}$selection (a) and a missing mass spectrum of $K^{+} \pi^{-}$with $K^{* 0}$ selection (b).

\section{LEPS II experiment}

\subsection{Introduction}

In this section, the status of development of a new $\mathrm{GeV}$ photon beam line at SPring-8, LEPS II, is described. The physics motivation for the LEPS II is described first, followed by the status report of the construction of LEPS II. The current LEPS is optimized to detect particles produced at forward 


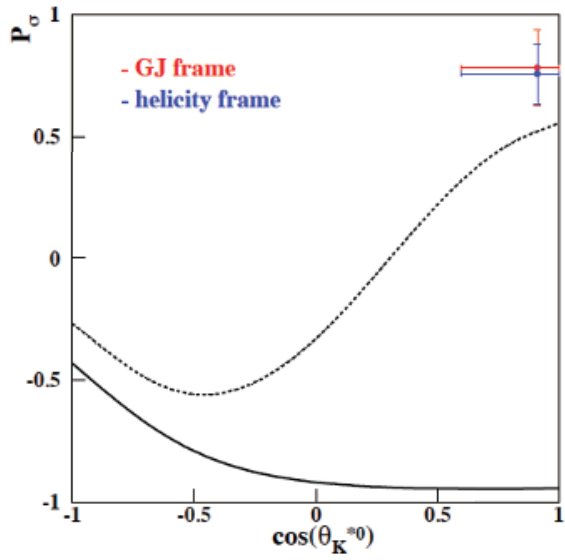

Fig. 2. Parity spin asymmetry $\left(P_{\sigma}=2 \rho_{1-1}^{-1}-\rho_{00}^{1}\right)$ in the helicity frame. The data point is averaged over photon energies from 1.85 to $2.96 \mathrm{GeV}$. The solid (dashed) line is the result of model I (model II) of Ref. [6] at $E_{\gamma}=$ $2.5 \mathrm{GeV}$. Model I has almost no contribution from $\kappa$ exchange, whereas model II includes substantial $\kappa$ exchange.

angles, and the acceptance for particles scattered at large angles is limited. The LEPS has reported various interesting data on the hadron photoproduction measured in the forward region [8]. One of the most important motivation to construct LEPS II is the search for a pentaquark candidate, $\Theta^{+}$. The LEPS collaboration reported the evidence of $\Theta^{+}$[9], in contrast, the CLAS collaboration observed no signal [10]. The inconsistent results of the LEPS and CLAS could be explained by the angular dependence of the production cross section because the LEPS detector covers very forward angle region where CLAS experiment doesn't have sensitivity. If the signal of $\Theta^{+}$is confirmed by LEPS in new data with higher statistics, in order to explain the null result of CLAS, it is indispensable to measure the production cross section in wide angle region.

One of the other motivations for LEPS II is to investigate the internal structure of $\Lambda(1405)$, which is a candidate of a meson-baryon molecule. Recent theoretical calculations with chiral Lagrangian and coupled unitary method predict that the mass peak of $\Lambda(1405)$ differs for its $\bar{K} N$ and $\Sigma \pi$ component [11]. It is important to investigate this two pole structure for the understanding of the internal structure of $\Lambda(1405)$ and the $\bar{K} N$ interaction. Hyodo et al. calculated the $\Lambda(1405) K^{*+}$ production off proton with linearly polarized photons. Similarly to the $\kappa$ meson search described in the previous section, the spin observables of $K^{*+}$ production can be used as the parity filter for the exchanged particle in the $t$-channel. The $K^{-}$exchange in the $t$-channel can be identified from the negative value of the parity spin asymmetry. The negative parity spin asymmetry represents the magnetic coupling of $g_{\gamma K^{*+} K^{-}}$, and as a result, the decay angular distribution of $K^{*}$ becomes perpendicular to the polarization angle of photons. Namely, we can select $K^{-}$exchange reaction by selecting events where $K^{*+} \rightarrow K \pi$ decay is perpendicular to photon polarization. Thus, Hyodo et al. predicted that the lineshape of $\Lambda(1405)$ changes to have $\sim 20 \mathrm{MeV}$ higher mass peak than PDG value when $K^{-}$exchange is selected. In order to reconstruct both $K^{*}$ and $\Lambda(1405)$, it is necessary to detect all decay products with a large solid angle detector. The LEPS II experiment is adequate for this measurement, since we can measure $K^{*} \Lambda(1405)$ production with a large solid angle detector and linearly polarized photons.

\subsection{LEPS II beam line}

The design and status of the construction of LEPS II beam line are described in this section. Figure 3 shows a top view of the LEPS II beam line. The laser photons are injected from the laser room toward the storage ring of the SPring-8. We use one of four long straight sections of SPring-8, BL31LEP, for LEPS II. The backward Compton scattering (BCS) of laser photons to 8-GeV electrons takes place in the straight section, and the scattered photons are irradiated to the target at $150 \mathrm{~m}$ downstream from 
the BCS collision point. Thanks to the low angular divergence of the electron beam in the long straight section, the BCS photon beam is very narrow, and the beam spot size at the target is about a few millimeters in RMS.

The maximum energy of BCS photons is $2.96 \mathrm{GeV}$ when a $266-\mathrm{nm}$ laser is used. In order to increase the intensity of BCS photon beam, photons from four laser modules are injected simultaneously. The long straight section of BL31LEP is advantageous to avoid the interference of lasers by changing the focus points of lasers along with the electron beam. In addition, further improvement of the luminosity of BCS photon beam is achieved by increasing the collision probability of a laser photon with a $8 \mathrm{GeV}$ electron. The electron beam has ellipsoidal cross section due to the bending magnets in the storage ring. Cylindrical lenses are used to shape the cross section of the laser beam to be ellipse, and the collision rate of laser photons to electrons gains twice. The beam intensity is $10^{6} \mathrm{cps}$ for $1.5-3.0 \mathrm{GeV}$ with the 266-nm Ar laser and 107 cps for 1.5-2.4 GeV with the 351-nm solid state laser, respectively. These are ten times higher than those of the LEPS I. The energy of a BCS photon is obtained by measuring the momentum of the recoiled electron. The scattered electrons are bended in the bending magnet of the storage ring more strongly than circulating $8-\mathrm{GeV}$ electrons. Thus, the momentum of the scattered electron is measured from the position of the electron after the magnet, and the energy of the BCS photon is obtained. The laser photons are highly polarized (higher than $95 \%$ ), and the $\mathrm{BCS}$ process conserves the polarization of photons in head on collision. As a result, $\mathrm{GeV}$ photon beam is also polarized higher than $90 \%$ depending on the energy. Thus, the hadron production experiment with high intensity and highly polarized photon beam will be possible in LEPS II.

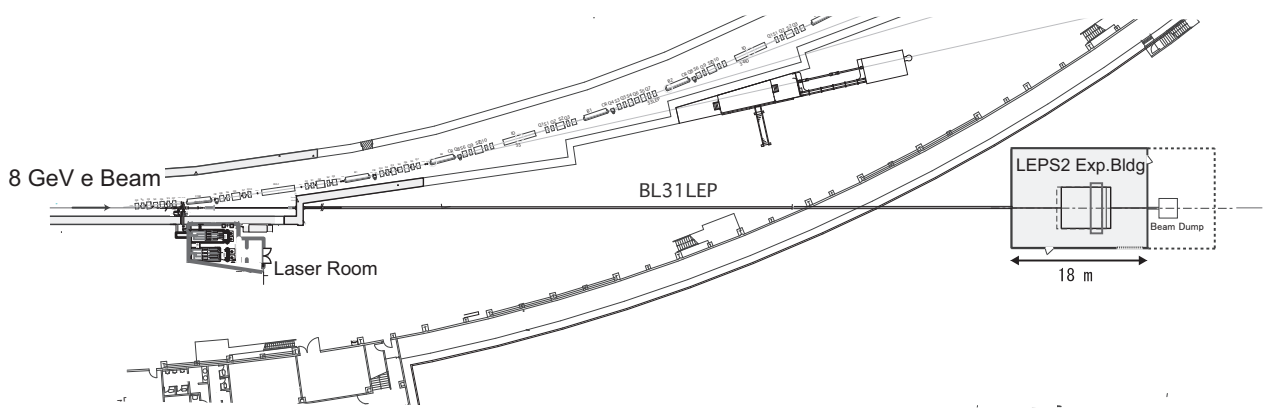

Fig. 3. Top view of the LEPS II beam line.

\subsection{LEPS II detector}

Figure 4 shows a schematic view of the LEPS II detector. The LEPS II detector is designed to measure both charged particles and photons simultaneously. A solenoid magnet for LEPS II is shipped from USA to Japan in 2011, which was used for the kaon rare-decay experiment at BNL-AGS, E787/E949 [12]. The inner volume of magnet is 2.22-m long and 2.96-m in diameter. The LEPS II detector will be installed in the solenoid magnet, which consists of double-sided silicon strip detectors (DSSD), forward drift chambers (FDC), a time projection chamber (TPC), time of propagation counter (TOP), resistive plate chamber (RPC-TOF), barrel electromagnetic calorimeter (EMCAL), and aerogel Čerenkov counter (AC). Charged particles are momentum-analyzed by the magnetic field of $1 \mathrm{~T}$. Charged particles scattered at forward angles are detected by the DSSD and FDC. On the other hand, the TPC measures the momentum in the large angle region. The signal of $K^{-} \Theta^{+}$production reported by LEPS is strong in the forward region, where $5<\theta_{K^{-}}<15$ degrees. Therefore, the momentum measurement at forward angles is required. The momentum resolution of $\sim 1 \%$ is achieved for $1 \mathrm{GeV}$ kaons in the range of polar angle $\theta>10^{\circ}$. The energy and direction of a photon are measured 
with EMCAL, which is shipped from BNL-AGS. The EMCAL is a sampling lead/plastic scintillator calorimeter with the total radiation length of $14.3 \mathrm{X}_{0}$ [12]. The polar angle coverage of the EMCAL is from $30^{\circ}$ to $110^{\circ}$.

The particle identification (PID) of charged particles are performed by TOF method in the low momentum region and by Čerenkov counter in high momentum region. A start signal for the TOF measurement is provided by the RF signal from the SPring- 8 storage ring, which has a time resolution of $12 \mathrm{ps}$. Since the speeds of a laser photon and a $8 \mathrm{GeV}$ electron are same, the arrival time of the BCS photon at the target is synchronized with the RF signal. A stop signal is provided by the RPC-TOF with $\Delta t \sim 50 \mathrm{ps}$, which is located at the barrel part where the radial distance from the target is about $1 \mathrm{~m}$. The good time-of-flight measurement provides $3 \sigma K \pi$ separation up to $1.1 \mathrm{GeV} / \mathrm{c}$. On the other hand, in the higher momentum region than $1.1 \mathrm{GeV} / \mathrm{c}$, Čerenkov counters are employed for PID. The high momentum particles up to $3 \mathrm{GeV} / \mathrm{c}$ are scattered at the forward region. The TOP counters are used to separate kaons from pions with $3 \sigma$ in the end-cap region [13]. The TOP counter is made of a quartz bar, which reconstruct the Čerenkov angle using the timing and position information of the Cerenkov photons traveling in the quartz bar. In addition, threshold type AC counters are used at the barrel part for the $K / \pi$ separation. The $e^{+} e^{-}$pair production is vetoed at the trigger level using the ultra low index $\mathrm{AC}$ with $n=1.008$. The trigger rate of hadron production is estimated to be $\sim 5 \mathrm{kcps}$, and a dead-timeless data acquisition system will be developed. The beam commissioning starts in early 2013, and the physics data taking will begin from 2013.

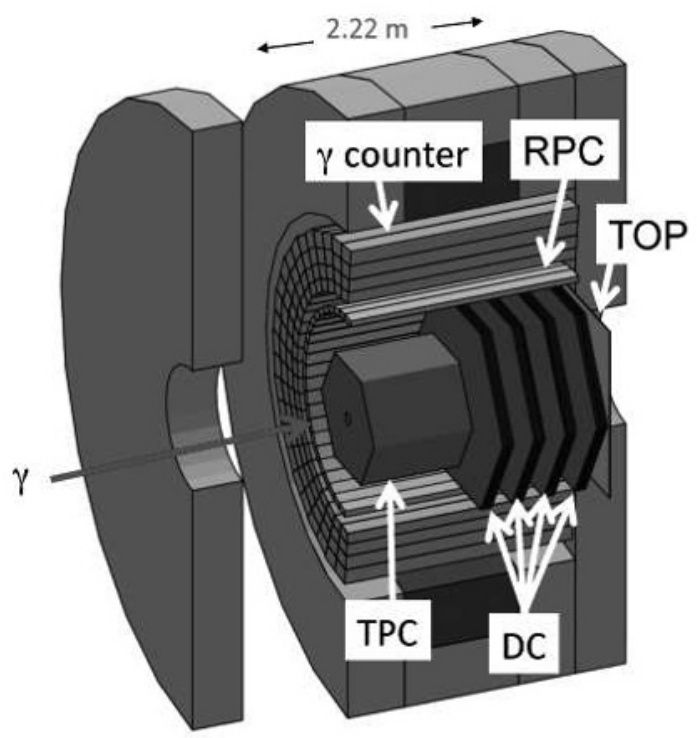

Fig. 4. Schematic view of the LEPS II detector.

\section{Summary}

In this report, the recent result of LEPS and the prospects of LEPS II have been reported. Firstly, the measurement of the parity spin asymmetry of $\gamma p \rightarrow K^{* 0} \Sigma^{+}$reaction was described in detail. The large positive value of $P_{\sigma}$ is consistent with a theoretical model with substantial contribution of $\kappa$ meson. Secondly, the status of the construction of a new $\mathrm{GeV}$ photon beam line at SPring-8, LEPS II, has described. The beam intensity will be improved one order of magnitude compared to the current LEPS. The development of a large solid angle detector which can detect both photons and charged 
particles is underway. The beam commissioning starts in early 2013, and the physics data taking will begin from 2013.

\section{References}

1. J. Beringer et al. (Particle Data Group), Phys. Rev. D86, (2012) 010001.

2. E. M. Aitala et al., Phys. Rev. Lett. 89, (2002) 121801.

3. A. Poluektov et al. (Belle Collaboration), Phys. Rev. D81, (2010) 112002.

4. S. H. Hwang et al. Phys. Rev. Lett. 108, (2012) 092001.

5. T. Mart and C. Bennhold, Phys. Rev. C 61, (1999) 012201.

6. Y. Oh and H. Kim, Phys. Rev. C 74, (2006) 015208.

7. T. Nakano et al., Nucl. Phys. A670, (2000) 332; A721, (2003) C112.

8. T. Mibe et al. Phys. Rev. Lett. 95, (2005) 182001.

M. Niiyama et al., Phys. Rev. C 78, (2008) 035202.

M. Sumihama et al. Phys. Rev. C 80, (2009) 052201.

9. T. Nakano et al. Phys. Rev. C 79, (2009) 025210.

10. B. McKinnon et al. Phys. Rev. Lett. 96, (2006) 212001.

11. T. Hyodo, A. Hosaka, M.J. Vicente Vacas, E .Oset Phys. Lett. B593,(2004) 75.

12. M. S. Atiya et al., Nucl. Instrum. Meth. A 321, (1992) 129.

13. M. Akatsu et al., Nucl. Instrum. Meth. A 440, (2000) 124. 